In proceedings of the 4th International Symposium on Distributed Objects \& Applications (DOA) pp. 693-710, Irvine, California, October 28 - November 1, 2002

\title{
Object Security Attributes: Enabling Application-specific Access Control in Middleware
}

\author{
Konstantin Beznosov \\ Quadrasis, Hitachi Computer Products (America), Inc., Waltham, MA \\ konstantin.beznosov@quadrasis.com
}

\begin{abstract}
This paper makes two primary contributions toward establishing support for application-specific factors in middleware security mechanisms. First, it develops a simple classification framework for reasoning about the architecture of the security mechanisms in distributed applications that follow the decision-enforcement paradigm of the reference monitor. It uses the framework to demonstrate that the existing solutions lack satisfying tradeoffs for a wide range of those applications that require application-specific factors to be used in security decisions while mediating access requests.

Second, by introducing attribute function in addition to decision and enforcement functions, it proposes a novel scheme for clean separation among suppliers of middleware security, security decision logic, and application-logic, while supporting application-specific protection policies. To illustrate the scheme on a concrete example, we describe its mapping into CORBA Security.
\end{abstract}

\section{Introduction}

The employment of application-specific factors in security decisions is not new, one example being target ADI in OSI access control framework [1]. Most current commercial distributed application security systems [2-5] provide various levels of support for application-specific information in policy decisions. What is missing, though, is a systematic inclusion of the support for these factors in the architecture of access control and other security mechanisms of middleware systems.

This void results in a lack of adequate architectural provisions in middleware security, leading to ad-hoc solutions. Although some efforts are under way to develop support for object security metadata in authorization policies [6], the present analysis did not reveal any notable in support of application-specific factors in the security mechanisms of distributed applications. Systematic support for application-specific factors is necessary, but it is missing in the architecture of distributed applications.

In this paper, we propose a schema for systematic support of application-specific factors in security mechanisms for distributed applications. To facilitate analysis of the existing solutions for supporting such factors, as well as to describe the proposed solution, we develop a framework for reasoning about those security mechanisms in distributed 
applications that follow decision-enforcement paradigm, e.g. access control, audit, and quality of message protection. The framework allows for classifying all solutions into four major schemes.

We use the framework to introduce our solution, which has two primary components: 1) generic representation of application-specific security-related factors in the form of object security attributes and 2) additional function for retrieving them at the time of access request mediation by the security sub-system. Being conceptually simple, the approach enables the use of application-specific factors in security policy decisions without coupling evaluation engines and target objects, while maintaining underlying middleware security application-neutral. Therefore, the security decision function can be provided by a third-party, while the enforcement function stays in the middleware, thus freeing the application owner from implementing either. To substantiate a relatively abstract explanation of the proposed solution, we show its translation into a concrete architecture of CORBA Security.

The reminder of the paper is organized as follows. The problem to be addressed is stated in Section 2. The framework is introduced and the available solutions are discussed in section 3. section 4 presents our solution in generic form. Its concrete application to CORBA Security is discussed in section 5. Discussion is provided in section 6 and conclusions are drawn in section 7 .

\section{Problem Motivation}

The problem raises because of the conflict of the following forces. On the one hand, a capable implementation of middleware security typically involves a complex and expensive piece of machinery, and is somewhat similar in its generality to operating system security. As a consequence of its critical nature, middleware security needs to be carefully designed, implemented, tested, assured, and tuned for performance and scalability. It is thus essential for the producers of middleware security to avoid alterations to their products and yet apply them to diverse application domains.

On the other hand, there is strong and natural interest among owners of distributed applications in making security decisions, mainly authorization ones, based on factors specific to the applications and organizational workflow, which is sometimes referred as object security metadata, as in Bonatti et al. [6].

\subsection{What are Application-specific Factors}

Unlike a resource security attribute, an application-specific factor is a certain characteristic or property of an application's resource, produced, modified and processed in the course of normal application execution and not for the sole purpose of a security policy decision. In OO middleware and other distributed systems, application objects are such resources. A remote analogy is resource access control decision information (ADI), defined as a description of the resource's security-relevant properties, in an ISO access control framework [1]. The difference between ADI and application-specific factors is that the former could be administered and utilized for the sole purpose of making access control decisions. 


\subsection{Examples}

To illustrate the needs of user organizations, consider the following real-life examples from the banking and telecommunication domains. Several people could be associated with each bank account, each having different rights. For example, the primary holder can do everything, including deleting the account, whereas the secondary holders, depending on the loyalty of the primary holder, could have different levels of limited access, such as withdrawing limited amounts and reviewing activities. All others can only deposit to the account. Implementation of such policies requires dynamic evaluation of the relationship between the accessing subject and an account. A list of account holders and their "rank" (e.g. primary and secondary) are such application-specific factors.

Security policies that U.S. long distance telephone carriers need to enforce depend on the state (e.g. Florida, Pennsylvania) in which a particular account is located. At the same time, accounts change phone numbers (and therefore possibly state) due to the relocations of their owners. Appearing to be a small and relatively infrequently occurring task, manually re-associating an account object with the corresponding state's policy becomes a resource-consuming operation for carriers with millions of subscribers (relocating, on average, every 5 years over 5,000 per day for $10^{6}$ subscribers). Instead, the policy could be determined using the first 6 digits of the phone number, which becomes one of the application-specific factors to be used in security policy decisions.

\subsection{Objective}

Also advocated by others [6] and identified as one of the input types for access decision function in [1], this demand for the use of application-specific information in authorization and other security decisions has a clear rationale. The more application or workflow information is used in security decisions, the better is the integration between security and application administration, which leads to low administration costs and fewer errors of application and security administrators. More importantly, enforcement of application-specific policies in the middleware frees developers from coding such policies in their applications, thus making systems less complex, quicker to build, and easier to evolve. These two factors result in significant long-term savings for application developers and owners.

At the same time, a number of enterprise-scale authorization products first appeared on the market of web servers security, and then expanded into application servers. These systems are good candidates for providing authorization services to distributed applications and the underlying middleware security. However, as we will show in the next section, there are technical obstacles in integrating them with both the former and the latter.

A question arises as to whether there a way to keep middleware security services generic and yet allow for enforcement of security policies specific to different application domains, possibly with the use of enterprise authorization systems.

\section{Available Solutions}

For the purpose of analyzing solutions to the problem, we differentiate all approaches to security policy decisions and enforcement in distributed systems based on two fac- 
tors: the nature of policy decision and enforcement functions. Roughly, each of these functions can be provided by the distributed application itself or by the security subsystem of the underlying distribution infrastructure, i.e. middleware security. Using acro-

Table 1: Acronyms for different locations of security policy decisions and enforcement

\begin{tabular}{|l|c|c|}
\hline & $\begin{array}{c}\text { Decision } \\
\text { Function }\end{array}$ & $\begin{array}{c}\text { Enforcement } \\
\text { Function }\end{array}$ \\
\hline \hline Middleware & $\mathrm{MD}$ & $\mathrm{ME}$ \\
\hline Application & $\mathrm{AD}$ & $\mathrm{AE}$ \\
\hline
\end{tabular}

nyms defined in Table 1, we have four possible combinations of decision and enforcement: MDME, ADME, ADAE and MDAE. In the following subsections, we explain each scheme and use this classification to assert that the available solutions do not provide desirable trade-offs.

\subsection{MDME -- Everything is done by Middleware}

The first case, the most obvious, occurs when both functions of security decision and its enforcement are provided by the middleware security, as shown in Figure 1. This is

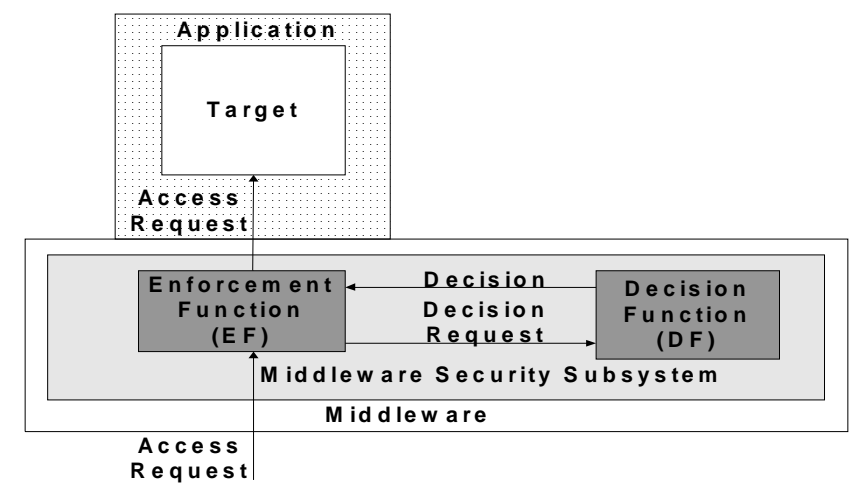

Figure 1 MDME schema

what practical middleware security systems implement. Being generic, both decision and enforcement functions come with the security subsystem. Applications are security-unaware, and therefore, are easier to design, develop, test, deploy and support. In addition, since the reference monitor is not "spilled" over into the application layer, assurance efforts are limited to the middleware layer and those below. This is why the MDME schema is considered to be the best for the purpose of enterprise security integration. However, with this approach no application-specific factors can be used for se- 
curity policy decisions. Consequently, the MDME schema is of no use for addressing the stated problem.

\subsection{ADME -- Application Decisions are Enforced in Middleware}

A better way is to externalize application-specific security logic into a separate service or module and make the middleware security subsystem to obtain policy decisions from it. This ADME schema is illustrated in Figure 2.

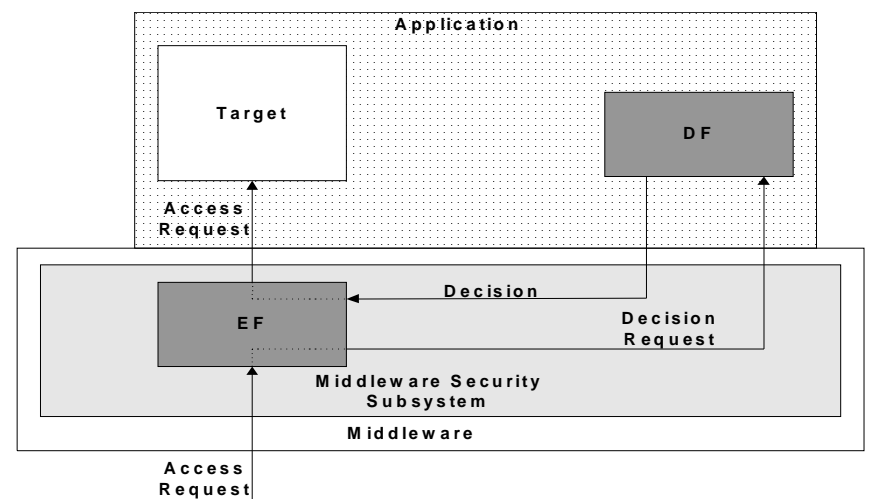

Figure 2 ADME schema

The schema has been realized in a number of middleware architectures. For example, CORBA Security [7] has replaceable AccessDecision and other interfaces. Java authentication and authorization service (JAAS) [8, 9], which recently became a part of J2SE v1.4, has replaceable interface Policy that serves authorization decisions. Although appearing to be versatile, the ADME schema comes with two major drawbacks.

Firstly, even being application-specific, the decision function (DF) still has the same generic interface for the enforcement function (EF) to query it because the latter remains generic. The interface protocols are not capable of communicating application-specific information between EF and DF. For instance, operation access_allowed() in CORBA's replaceable AccessDecision interface takes subject's credentials, reference to the target object (just "target" for short), target type, and operation on it. Java's Policy::getPermissions() accepts, as input parameters, information representing the original location of the code and the public keys of its signer. Microsoft's .NET security model, although not currently well documented, appears to have a security architecture similar to Java in this regard. Clearly, neither of them provide for application-specific factors to be communicated from enforcement to decision point.

And, even if the DF interface supported communication of application-specific factors, the means of retrieving such factors in the EF are not defined. Thus, a custom implementation of DF would have to employ a back door to go back to the target object (or a data repository) and retrieve application-specific factors from there, as shown in Figure 3.

When a back door is provided by a target object, the main drawback of the technique is due to the performance hit. Specifically, in some applications, it could be expensive to 


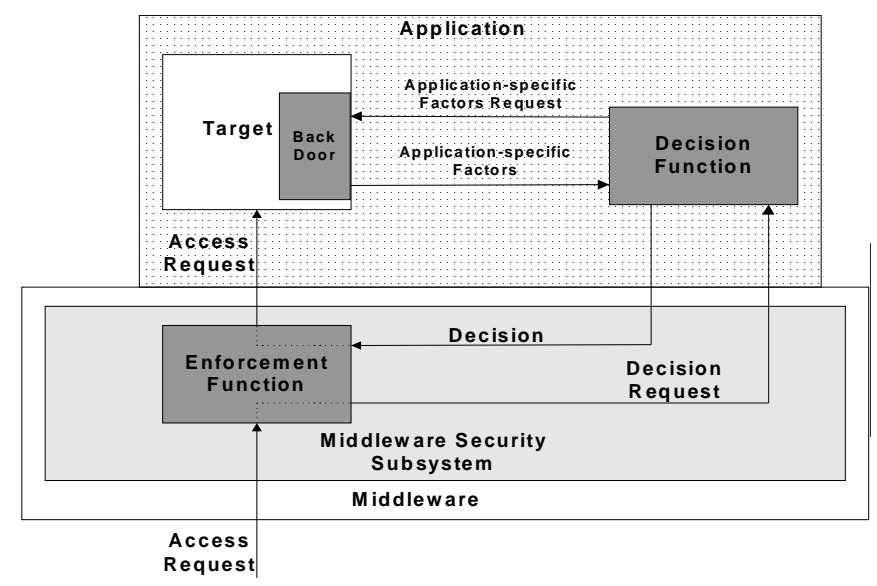

Figure 3 Back Doors to Target in ADME Schema

restore the target objects' state and make them ready to serve requests, including those coming through back doors. Consider applications that use secondary or even tertiary storage for the object state between sessions. It could be prohibitively expensive to restore an object, obtain some data through its back door, and make an authorization decision just to find that access has been denied. Performing expensive re-incarnation of target objects for making security decisions also creates a vulnerability for denial of service attacks.

Despite DF having a potential to be specific to the application domain, ADME schema allows enforcement of only those run-time pre-requisites, such as (dynamic) conditions in [10, 11] and [6], obligations in XACML [12] and provisions in [13], that are non-specific to application domains (such as CPU load). This limitation is due to EF being part of the generic middleware layer.

Secondly, being an all-or-nothing solution, the use of the application-specific decision function forces the new logic to re-implement authorization decisions completely, which is prohibitively complex and difficult to do correctly for distributed large scale systems, thus rendering this approach unrealistic for most user organizations with the needs in application-specific authorization and other security logic. End-user organizations do not want to be in the business of implementing authorization and other security policy evaluation engines, which is required if application-specific factors were to be used in security policy evaluation.

\subsection{ADAE -- Security-aware Applications}

ADAE schema is more flexible for making security decisions based on application-specific factors then the previous two. The intent is to let an application-provided EF to call the DF (also provided by the application), thus obviating the problem of obtaining application-specific factors by a generic EF. The schema is illustrated in Figure 4. Although generic, the protocol of supplying necessary information to a DF and retrieving decisions from it can be used for communicating application-specific factors. 


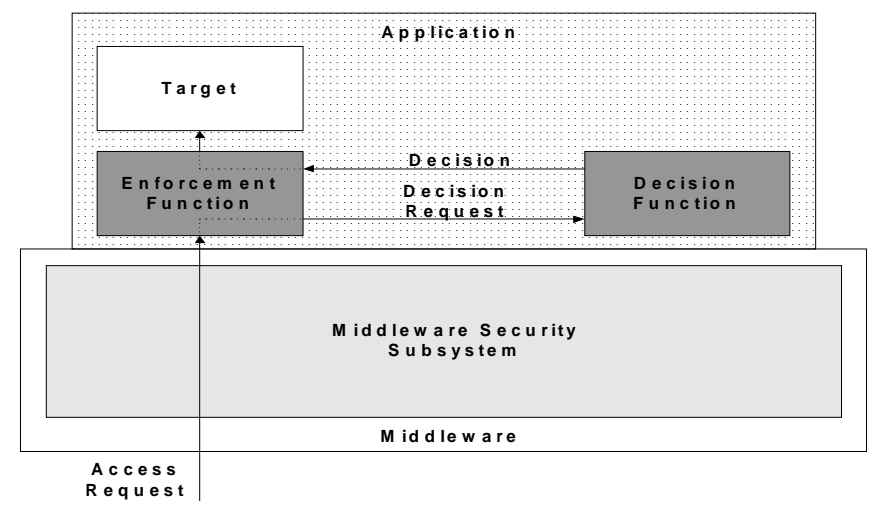

Figure 4 ADAE schema: general case

The general case (Figure 4) of this schema, when EF is external to targets, although being employed in some research systems [14-17], is not known to be popular in real-life solutions. We believe this is because it requires a proxy object implementing EF to "wrap" each target, and it does not allow enforcement of fine-grain policies because EF is outside of the target.

\subsubsection{ADAE With Target Implementing EF}

However, a particular case of ADAE -- when EF is implemented in the target -- as shown in Figure 5, is widely used. It is popular in distributed application systems con-

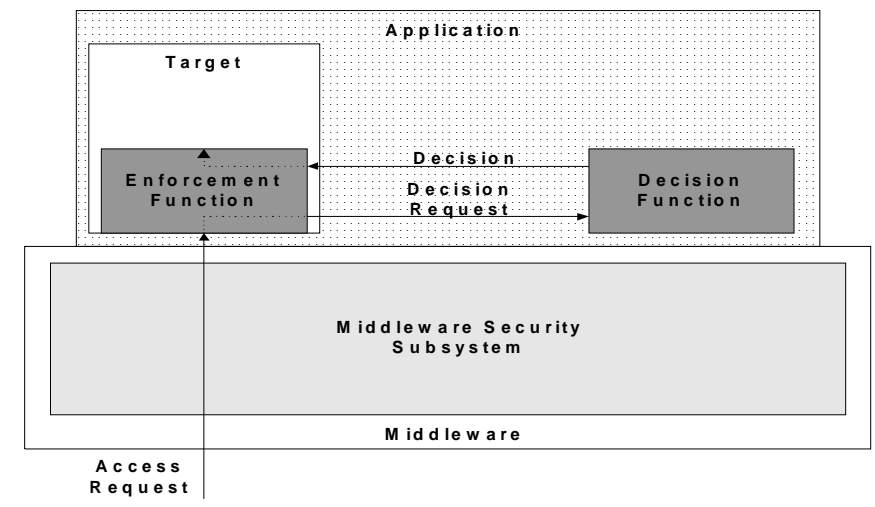

Figure 5 ADAE schema: target implements EF

structed on top of limited middleware security technologies, e.g., those lacking access control enforcement, such as Kerberos [18] and SESAME [19]) or implementations. Other reasons for employing ADAE schema with EF implemented in targets are the capability to enforce fine-grain security policies and the ease of obtaining application-specific factors because of the collocation of the business and enforcement functions. 
One example of the approach is Resource Access Decision (RAD) architecture [20, 21], where a resource name, composed of a name-value pair list, can be used for encoding application-specific factors. In our example with long distance telephone carriers, account's phone number, encoded in the name of the resource in question, can be now used during policy evaluation. ${ }^{1}$

Other examples of middleware security systems in which the enforcement function is implemented by an application system are Praesidium [22], Adage [23], GAA API [10, $11,24]$ and Access Control Unit in [6]. As in RAD, these solutions feature an authorization function invoked by an application for obtaining access control decisions, which are expected to be enforced by an application.

Unfortunately, this active role of the target in composing queries to DF and enforcing decisions results in a number of disadvantages, the most salient one being the necessity for security-related code to be mixed with business logic, thus making targets securityaware. As we argue in [25], this security awareness by target objects makes them more complex and prone to security vulnerabilities. It also makes it more difficult to perform security assurance, and forces application developers to be experts in security programming, despite externalizing security decision logic.

Being the best and sometimes the only viable solution for particular cases with complex, application-specific, or fine-grain security policies, programming EF inside of target objects is still a sub-optimal approach for those application domains where a combination of a general purpose security decision function with application-specific attributes could suffice.

\subsection{MDAE Schema}

We are not aware of any solutions that employ MDAE schema. This is not surprising, since application-executed enforcement of security decisions made by the middleware security subsystem does not seem to bring any advantage over any other scheme, while having all the disadvantages and limitations of MDME schema and some of ADAE.

\subsection{Real-Life Hybrids}

Several commercial solutions for securing web and middleware applications $[2-5,26]$ implement an authorization server. It can be queried by either an application itself (ASP or JSP in a web server, bean in EJB container, or CORBA/COM application object) or a middleware-specific enforcement function (web server filter, EJB container, or CORBA security interceptor). Although widely employed in large organizations, neither of these usages introduces any novel solution to the stated problem. The former is an instance of ADAE and the latter, ADME schemas.

\section{Generic Solution -- ADME/AF Schema}

We introduce a new approach, here, that enjoys the advantages of ADME schema and yet enables the use of application-specific factors in security policy decisions without coupling evaluation engines and target objects. Therefore, DF can be provided by a

1. Clearly, this requires appropriate programming of the decision function. 
third-party company, including an authorization product vendor, while EF stays in the middleware, thus freeing the application owner from implementing either.

As we discussed in section 3.2, though allowing security decision logic to be application-specific, the original ADME schema suffers from a lack of the means: a) to communicate application-specific factors to DF and, most importantly b) to obtain them, given the target object in question.

We address the first, simpler, problem by introducing generic representation for application-specific factors. These, object security attributes (OSA), as we refer to them, can be expressed in a number of formats, varying in complexity from name-value pairs to arbitrary XML-based structures. The semantic interpretation of an OSA is completely up to the processing entity -- DF. In our example with a telecommunication carrier, for each account object there could be an OSA "holding" the current phone number of the account. More than one OSA can be associated with a target object, comprising a collection of OSAs.

We resolve the second issue, obtaining OSAs for the corresponding target object, by introducing additional function in the ADME schema -- the attribute retrieving function, or attribute function (AF), as shown in Figure 6. This function has simple syntax: it ac-

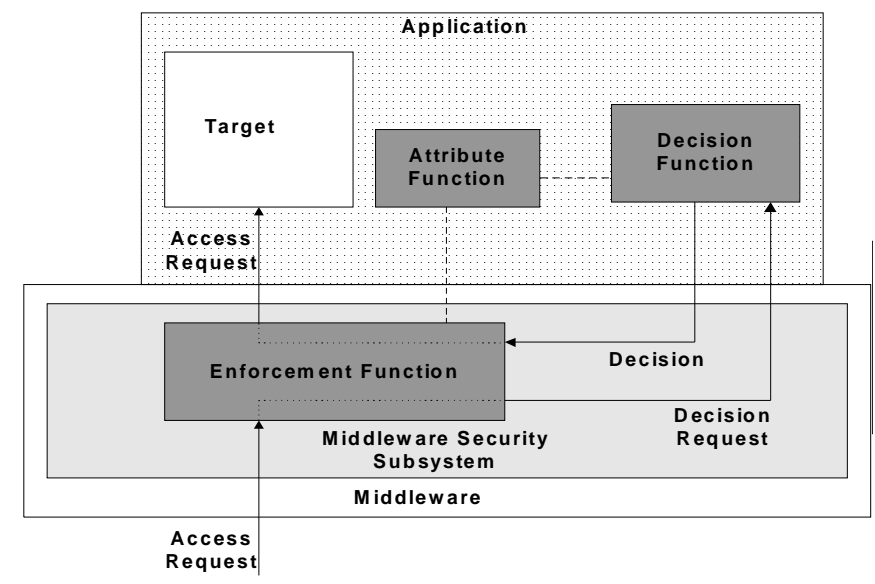

Figure 6 Attribute function in ADME schema

cepts (middleware-specific) data that are necessary for identifying the state of the target object and returns a set of OSAs for that object. The target object state is necessary for retrieving such object metadata as its OSAs. Since OSA semantics are very specific to the application being protected, AF is provided by the application and not by the middleware or security layers.

The function that obtains OSAs via AF is very dependent on the particular implementation of the approach. In some circumstances, EF could be in better position to make an invocation to AF; in others, DF could be in a better position to do so. Moreover, some implementations could make EF and DF to perform this step together. For example, in 
section 5, we show how CORBA-specific realization of ADME/AF schema splits AF into two objects, one of which is invoked by EF and the other by DF.

There are a number of advantages if AF is invoked by EF. First, data, necessary for identifying the target state that $\mathrm{EF}$ has at its disposal at the invocation point, are very specific to the middleware technology and the type of the particular object adapter that hosts the target. Therefore, for a DF to obtain OSAs from AF, EF would have to pass such data to DF. Second, since DF is usually a COTS, which serves authorization decisions in other schemas, such as ADAE, and for different middleware systems, decision interface to it is too generic to support target state data. Third, there could be more than one DF -- one for each type of security policy, e.g., authorization, audit, quality of protection, and non-repudiation -- invoked at every access to a target. It seems beneficial to minimize the number of potentially expensive invocations on AF by obtaining OSAs once per access request.

On the other hand, postponing invocation of AF allows for lazy strategy, i.e., OSAs are retrieved only if some DF is going to use them. Moreover, a DF could require only particular OSA(s). Retrieving only needed OSA(s) is simple to implement when invocation to $\mathrm{AF}$ is done by $\mathrm{DF}$.

This introduction of OSAs as a way to represent target metadata related to security decisions in distributed applications, and a function for retrieving them, $\mathrm{AF}$, enables security decisions to be application-specific, while keeping EF in the middleware security layer and using COTS authorization, as well as other security policy decision functions, systems without modifications. We refer to this approach as ADME/AF schema.

\section{Application to CORBA Security}

Thus far, we have stated the problem, analysed available solutions, and presented our approach in a form independent of any particular middleware technology. Further, we generalized the solution so that it is applicable not only to the access control, but also to other security functions that can be decomposed into decision and enforcement phases on each access request. Now, we move on to consider a particular middleware security technology -- CORBA security -- and demonstrate how the general solution applies to it.

\subsection{CORBA Architecture}

This section provides a description, adopted from [27, 28], of CORBA ORB architecture. The architecture consists of several primary components, as illustrated in Figure 7 and described below.

Target object or object -- a CORBA programming entity that consists of an adapterspecific identity, an interface, and an implementation, which is known as a Servant. Servant is an implementation programming language entity that defines the operations that support a CORBA IDL interface. Servants can be written in a variety of languages, including C, C++, Java, Smalltalk, and Ada.

Client -- the program entity that invokes an operation on an object implementation using object reference (OR). Accessing the services of a remote object should be transparent to the caller. Ideally, it should be as simple as calling a method on an object, i.e., 


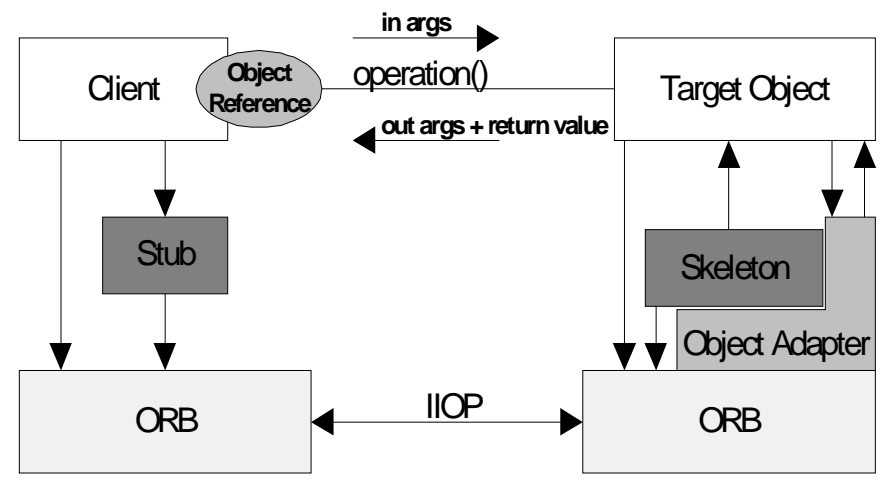

Figure 7 CORBA ORB architecture

obj->op(args). The remaining components in Figure 7 help to support this level of transparency.

Object Request Broker (ORB) provides a mechanism for transparently communicating client requests to target object implementations. It simplifies distributed programming by decoupling the client from the details of the method invocations, thus making client requests appear to be local procedure calls. When a client invokes an operation, the client and target ORBs are responsible for finding the object implementation, transparently activating it if necessary, delivering the request to the object, and returning any response to the caller. An ORB is a logical entity that may be implemented in various ways, such as through one or more processes or a set of libraries. To decouple applications from implementation details, the CORBA specification defines an abstract interface for an ORB, which provides various helper functions.

CORBA IDL stubs and skeletons serve as the "glue" between the client and server applications, respectively, and the ORB. The transformation between CORBA IDL definitions and the target programming language is automated by a CORBA IDL compiler. The use of a compiler reduces the potential for inconsistencies between client stubs and server skeletons and increases opportunities for automated compiler optimizations.

Object Adapter (OA) assists the ORB in delivering requests to the object and with activating the object. More importantly, an object adapter associates object implementations with the ORB. Object adapters can be specialized to provide support for certain object implementation styles, e.g., OODB object adapters for persistence and library object adapters for non-remote objects. The ORB and the OA cooperate to allow client applications to invoke requests on CORBA objects and to ensure that each valid CORBA object is mapped to a servant. In addition, the ORB and the OA cooperate to transparently locate and invoke the proper servants given the addressing information stored in CORBA object references.

The primary type of OA used in current CORBA applications is portable object adapter (POA) [29]. A server application can have multiple POAs nested within it. An application might want to create multiple POAs to support various types of CORBA objects and/or different kinds of servant styles. For example, the application might have two POAs, one that supports transient objects and one that supports persistent objects. 
A nested POA can be created by invoking a factory operation on another POA. All servers have at least one POA, the Root POA. To create a POA nested under the Root POA, the application invokes the create POA operation on the Root POA. The object reference for the Root POA is available from the ORB. The characteristics of each POA, other than the Root POA, are controlled at POA-creation time using different POA policies.

\subsection{Run-time CORBA Security}

CORBA Security service (CS) [7] defines interfaces to a collection of objects for enforcing a range of security policies using diverse security mechanisms. It provides abstraction from an underlying security technology so that CORBA-based applications can be independent from the particular security infrastructure provided by the user enterprise computing environment. Due to its general nature, CS is not tailored to any particular access control model. Instead, it defines a general mechanism that is supposed to be adequate for the majority of cases and could be configured to support various access control models. The CS model comprises the following functionalities, among others, visible to application developers and security administrators: identification and authentication, authorization and access control, auditing, integrity and confidentiality protection, authentication of clients and target objects, optional non-repudiation, and administration of security policies.

One of the objectives of CS is to be totally unobtrusive to application developers. Security-unaware target objects should be able to run securely on a secure ORB without any active involvement on their site. Meanwhile, it must be possible for security-aware objects to exercise stricter security policies than those enforced by CS. In the CS model, all object invocations are mediated by the appropriate security functions in order to enforce various security policies such as access control. Those functions are part of CS and are tightly integrated with the ORB and the corresponding OAs.

Security policies are enforced completely outside of an application system at the ORB level. Everything, including obtaining information necessary for making policy decisions, such as access control, is accomplished before the method invocation is dispatched to the target object. As Figure 8 shows, policy enforcement code is executed inside the CORBA Security enforcement sub-system, when a message from client application to a target object is passed through the ORB. Executed at the client ORB as well as at the target ORB, the enforcement code uses the following three sources of information for making policy decisions to enforce:

- The policy of the domain(s) to which the target belongs.

- The information from credentials of the client. In case of access control policy enforcement, these are client privilege attributes, such as access identity, group membership, roles and clearance. Whereas for audit policy enforcement, security attribute of type AuditId is used.

- The access request itself.

Although a CORBA security sub-systems can be, and usually is, integrated with the ORB and OAs using proprietary means, for the sake of simplicity, we conceptualize its enforcement function as a security interceptor. 


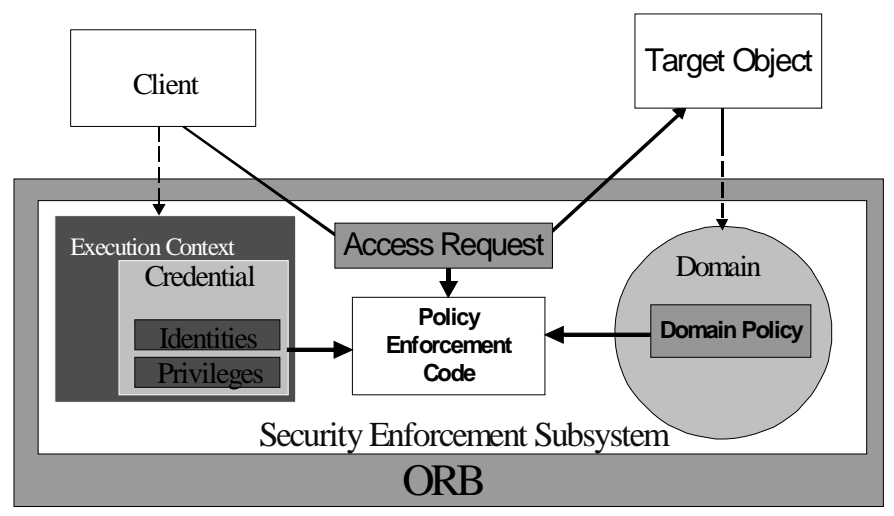

Figure 8 Enforcement of policies in CORBA Security subsystem

\subsection{Attribute Retrieval Function}

In our solution to the domain of CORBA Security, the closest analogy to AF is interface AttributeRetriever. This interface provides the following operations for retrieving OSAs:

- get_attributes_by_type(type: AttributeType ) : AttributeValueList

- get_all_attributes() : ObjectAttributeList

Having its operations implicitly tied into particular target object, the interface constitutes only part of AF, which is invoked by DF. However, there is another part of AF invoked by EF. This is due to the problem of invoking adapter-specific AF by adapterneutral DF. AF has to be specific to the type of object adapter because, as in most middleware technologies, the notion of object universal identity is not well developed in CORBA architecture. ${ }^{1}$ But object-specific identity is sufficiently strong in the context of a particular object adapter, i.e., the adapter has a sufficient amount of information to dispatch an access request to the right object servant, which is responsible for processing application requests for the object in question. On the other hand, it is highly undesirable to have decision functions specific to the adapter type.

This is why an additional level of indirection via interface Manager, the other part of $\mathrm{AF}$, has been introduced. Adapter-specific derivatives of Manager locate AttributeRetriever objects and return them to the EF, which is implemented in the form of a security interceptor. Being adapter-specific, such an interceptor takes control over the access requests, as well as obtains and enforces policy decisions. Before a security interceptor invokes DFs, it obtains a local reference to the correct AttributeRetriever object from the corresponding Manager, and places it on Current object that serves as a thread-specific placeholder for OSA-related information. Later, when the interceptor calls AccessDecision and other objects, their implementations can obtain OSAs from AttributeRetriever via its OR available off Current object. This lazy strategy of making AttributeR-

1. See [30] for detailed discussion on the shortcomings of object identity in middleware security. 
etriever available for subsequent queries by DFs allows for retrieval of OSAs only if necessary. If OSAs are required more than once per access request, an AttributeRetriever could cache results of time expensive retrieval operation.

\subsection{Registering and Discovering Managers}

Another issue involves the means for an application to register and for the interceptor to obtain a local reference to a Manager. Along with this, an additional issue required a resolution, specifically what should be the scope of Manager, i.e., should one Manager serve AttributeRetrievers for all objects in a given application. And, if not, then how should a security interceptor determine what Manager serves a given object? We saw certain benefits in making the solution flexible and supporting existence of several Managers for each application. This flexibility allows various implementations of AttributeRetrievers in one application. Moreover, since adapter-specific derivatives of Manager must be used, the limitation to only one Manager instance would prevent different object adapters from co-existing in the same application.

However, it turned out to be difficult to find a way to share multiple Managers among process collocated objects. The essence of the problem is the lack of a placement for the information associating an object with a Manager. An object adapter appeared to be the only appropriate place to store such information with good chances to retrieve it efficiently at the time of mediating an access request. The associating information is stored in the form of ManagerPolicy. This lightweight object holds a reference to the Manager instance. The use of ManagerPolicy follows the design philosophy exercised in the design of POA, where configuration of a particular POA instance is encoded in the form of POA policies "attached" to the adapter at the time of its creation.

The relationships between target objects, Managers, ManagerPolicies and object adapters is illustrated in Figure 9. Access requests for any given object are originally

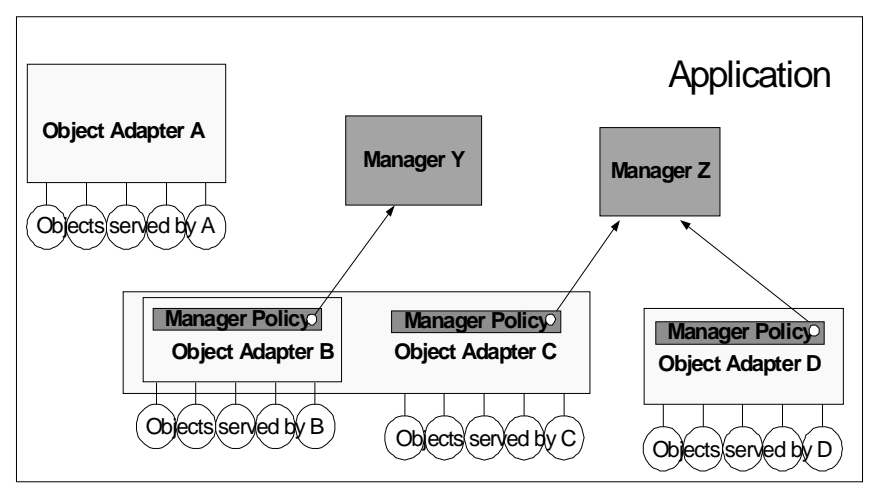

Figure 9 Relationships among objects, object adapters, OSA Managers, and ManagerPolicies

pre-processed and then dispatched by the corresponding object adapter $(\mathrm{OA})$ to the object's servant. OAs can constitute hierarchies with one root, unlimited child and corresponding parent adapters. For each OA, there could be no more than one Manager that 
serves OSAs for all objects under the adapter. That Manager is said to serve the OA. The same Manager can serve more that one OA, as in the case of object adapters $\mathrm{C}$ and $\mathrm{D}$ and Manager $\mathrm{Z}$, as seen in Figure 9.

Each OA could contain an instance of ManagerPolicy, which holds an object reference to the Manager serving the adapter. If an application provides a Manager for the given OA, it sets ManagerPolicy (containing a reference to the Manager) on the adapter using mechanisms specific to the adapter type. For example, such a policy, among others, is passed to the adapter's parent at the child's creation time in the case of POA. If no ManagerPolicy is set on an OA, as for adapter A (Figure 9), then no Manager serves this adapter, which is equivalent to the lack of OSAs associated with the adapter's objects. The diagram in Figure 10 depicts a sequence of invocations that an application performs

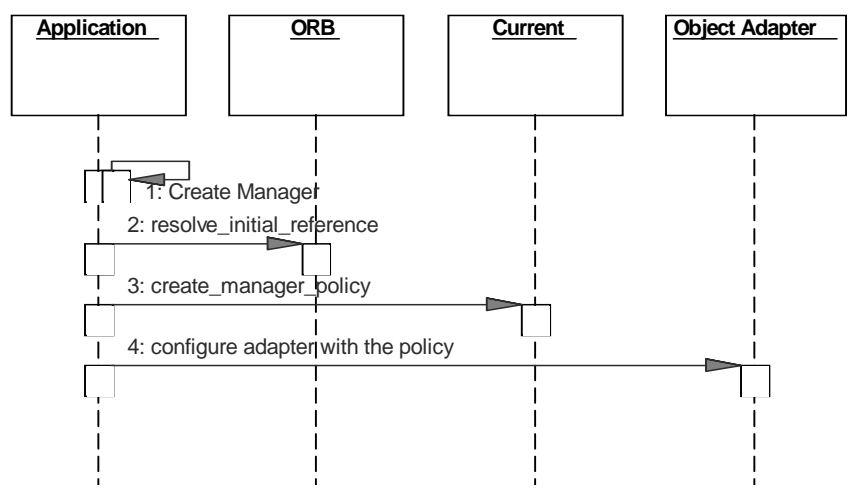

Figure 10 Sequence of Steps Done by an Application for Registering a Manager

for registering its Manager implementation with an OA. After creating an implementation of adapter-specific Manager sub-interface (invocation 1 in the sequence diagram), the application obtains a reference to the Current from the ORB by invoking ORB::resolve_initial_references() with argument "ObjectSecurityAttributeCurrent" (invocation 2). Then, the application uses a factory operation on the Current interface, to which it supplies a valid OR for the Manager, for creating an instance of a ManagerPolicy (invocation 3). While processing the invocation, Current creates an instance of ManagerPolicy and returns it to the caller.

The final step (invocation 4) is to "hand" the ManagerPolicy to the OA using the OAspecific mechanisms. In the case of POA, for example, an instance of ManagerPolicy is inserted, with other CORBA::Policy objects, in the list of policies provided to the parent POA as an argument of factory operation PortableServer::POA::create_POA() for creating the POA that will serve the same objects as the Manager referred in the ManagerPolicy will.

Detailed architecture of the solution in the realm of CORBA Security is provided in [31]. The work presented in this section became a CORBA standard adopted by the Object Management Group, and it is currently on finalization track. 


\section{Discussion}

ADME/AF schema is not the ideal and does not work for all cases. Fore examples, being derived from ADME, it would not work for applications that require fine grain protection, because its granularity is not finer than methods exposed by a target object. It also does not support application-specific pre-requisites.

Further, if a DF has to call an AF, then its implementation becomes specific to AF interface; yet additional DF wrapping could help. Some middleware technologies, such as $\mathrm{COM}+$ [32], do not provide mechanisms for implementing ADME schemas, which limits the applicability of this approach. Those rare applications in which security policies are difficult to express using application-specific factors, fall out of the applicability scope as well.

While having these limitations and disadvantages, the ADME/AF schema offers all the flexibility of embedding decision functions in the target without requiring the target to be security aware, for those applications whose security policies have moderate granularity requirements, can be expressed using OSAs, and need only decisions enforceable by generic EF.

Our solution enables the process of implementing secure distributed applications to be cleanly separated among:

- middleware security suppliers, who implement EF,

- security logic suppliers, who implement DF,

- application suppliers, who implement target objects, and possibly AF, and

- application owners who, having possibly AF implemented, configure EF, AF and DF to work together and enforce application-specific protection.

While we deliberately limited the proposed approach to the domain of distributed applications, it may be useful to investigate its applicability and utility for operating systems, as an example.

\section{Conclusions}

In this paper, we described the problem of supporting application-specific factors in security mechanisms in the field of distributed application systems. In order to address the problem, we first created a framework for reasoning about those security mechanisms in distributed applications that follow decision-enforcement paradigm. Employing the framework, we showed that all cases can be partitioned into one of four schemas, depending on whether middleware security or application provides decision and/or enforcement functions. Although offering the most promising solution for the stated problem, ADME schema nonetheless lacks the means of obtaining and communicating application-specific factors to DF. In the described solution, we introduced the notion of an object security attribute (OSA) as a generic way to represent security-related information about the application object being accessed. More importantly, we proposed attribute retrieval function (AF) that serves attributes to DFs, as a new element to the updated schema. By introducing ADME/AF schema, we made the initial step toward sys- 
tematic support for application-specific factors in middleware access control mechanisms.

To illustrate the concepts of OSAs and AF on a concrete technology, we described their realization for CORBA Security. The described application of ADME/AF schema to CORBA domain has been adopted by the Object Management Group as part of SDMM specification [31] in November 2001, and it is currently on finalization track.

\section{Acknowledgements}

The concept of object security attributes was prompted by a conversation with Ron Monzillo at an OMG TC meeting in 2000. Their specific architecture and realization for CORBA Security have their origins from the work on SDMM specification that has undergone numerous discussions. Some individuals who contributed most to these discussions and helped with their critiques of OSA architecture are Ted Burghart, Fred Dushin, Don Flinn, Bret Hartman, Polar Humenn, Tadashi Kaji, Jishnu Mukerji, Mindy Rudell and Kent Salmond.

Thanks to Bret Hartman for reviewing the paper in its early stage and for providing helpful comments.

\section{References}

[1] OSI, "Information Technology -- Open Systems Interconnection -- Security frameworks in open systems -- Part 3: Access control," ISO/IEC JTC1 10181-3, 1994.

[2] G. Karjoth, "The Authorization Service of Tivoli Policy Director," presented at Annual Computer Security Applications Conference (ACSAC), New Orleans, Louisiana, 2001.

[3] Netegrity, "SiteMinder Concepts Guide," Netegrity, Waltham, MA 2000.

[4] Entegrity, "Entegrity AssureAccess ${ }^{\mathrm{TM}}$ - Technical Overview," Entegrity Solutions, September 2000.

[5] Securant, "Unified Access Management: A Model For Integrated Web Security," Securant Technologies, June 25 1999, http://www.cleartrust.com.

[6] P. A. Bonatti, E. Damiani, S. D. C. d. Vimercati, and P. Samarati, "A Component-based Architecture for Secure Data Publication," presented at ACSAC, New Orleans, Louisiana, 2001.

[7] OMG, "CORBAservices: Common Object Services Specification, Security Service Specification v1.7," document formal/01-03-08 2001, http://www.omg.org/cgi-bin/doc?for$\mathrm{mal} / 01-03-08$.

[8] C. Lai, L. Gong, L. Koved, A. Nadalin, and R. Schemers, "User Authentication And Authorization In The Java Platform," presented at ACSAC, Phoenix, Arizona, USA, 1999, http://java.sun.com/security/jaas/doc/acsac.html.

[9] Sun, "Java Authentication and Authorization Service (JAAS)," Sun Microsystems, 2001.

[10] T. Ryutov and C. Neuman, "Access Control Framework for Distributed Applications (Work in Progress)," IETF, Internet Draft draft-ietf-cat-acc-cntrl-frmw-03, March 92000.

[11] T. Ryutov and C. Neuman, "Representation and Evaluation of Security Policies for Distributed System Services," presented at DARPA Information Servability Conference Exposition, Healton Head, South Carolina, 2000. 
[12] XACML-TC, "OASIS eXtensible Access Control Markup Language (XACML), Committee Draft," OASIS May 9 2002, http://www.oasis-open.org/committees/xacml/docs/.

[13] M. Kudo and S. Hada, "XML Document Security Based on Provisional Authorization," presented at ACM Conference on Computer and Communications Security, Athenes, Greece, 2000.

[14] B. Hailpern and H. Ossher, "Extending Objects to Support Multiple Interfaces and Access Control," IEEE Transactions on Software Engineering, vol. 16, pp. 1247-1257, 1990.

[15] J. Barkley, "Implementing Role-based Access Control Using Object Technology," presented at The First ACM Workshop on Role-Based Access Control (RBAC), Fairfax, Virginia, USA, 1995.

[16] R. Filman and T. Linden, "SafeBots: a Paradigm for Software Security Controls," presented at New Security Paradigms Workshop, Lake Arrowhead, CA USA, 1996.

[17] T. Riechmann and F. J. Hauck, "Meta Objects for Access Control: A Formal Model for Role-based Principals," presented at New Security Paradigms Workshop, Charlottesville, VA USA, 1998.

[18] IETF, "RFC 1510, The Kerberos Network Authentication Service, V5," Internet Engineering Task Force, 1993.

[19] T. Parker and D. Pinkas, "SESAME V4 - Overview," SESAME, December 1995.

[20] OMG, "Resource Access Decision Facility," OMG, document number: formal/2001-0401, August 2001, http://www.omg.org/cgi-bin/doc?formal/2001-04-01.

[21] K. Beznosov, Y. Deng, B. Blakley, C. Burt, and J. Barkley, "A Resource Access Decision Service for CORBA-based Distributed Systems," presented at ACSAC, Phoenix, Arizona, USA, 1999.

[22] HP, "HP Adds Value to DCE Security Framework with Praesidium Authorization Server," in DCE Application Development Trends Magazine, 1996.

[23] R. Simon and M. E. Zurko, "Adage: An Architecture for Distributed Authorization," OSF Research Institute, Cambridge 1997, http://www.osf.org/www/adage/adage-arch-draft/adage-arch-draft.ps.

[24] T. Ryutov and C. Neuman, "Generic Authorization and Access control Application Program Interface: C-bindings," IETF, draft-ietf-cat-gaa-bind-03, March 92000.

[25] B. Hartman, D. J. Flinn, and K. Beznosov, Enterprise Security With EJB and CORBA. New York: John Wiley \& Sons, Inc., 2001.

[26] Encommerce, "getAccess Design and Administration Guide," Encommerce, September 20 1999, http://www.encommerce.com.

[27] D. C. Schmidt, "Overview of CORBA," 2001, http://www.cs.wustl.edu/ schmidt/corbaoverview.html.

[28] D. C. Schmidt and S. Vinoski, "Object Adapters: Concepts and Terminology," in SIGS C++ Report, vol. 9, 1997.

[29] OMG, "Specification of the Portable Object Adapter (POA)," OMG document \# formal/ 01-09-48, 2001.

[30] U. Lang, D. Gollmann, and R. Schreiner, "Verifiable Identifiers in Middleware Security," presented at ACSAC, New Orleans, Louisiana, 2001.

[31] OMG, "Security Domain Membership Management Service, Final Submission," document \# orbos/2001-07-20, July 112001.

[32] K. Brown, Programming Windows Security, First ed. Upper Saddle River, NJ: AddisonWesley, 2000. 